\title{
Pattern of urinary tract infection in Kashmir and antimicrobial susceptibility
}

\author{
Ahmad S \\ Associate Professor of Medical Microbiology and Consultant Microbiologist, Department of \\ Clinical Laboratory Sciences, College of Medical Sciences, Salman Bin Abdul Aziz University, \\ Kingdom of Saudi Arabia, E-mail: drshamweel@hotmail.com
}

\begin{abstract}
Antibiotic resistance of urinary tract pathogens has increased worldwide. The purpose of this study is to provide information regarding the causative agents of urinary tract infection in Kashmiri patients, identify the uropathogens responsible for the infection and study the antibiotic susceptibility patterns of the uropathogens. Clean voided mid-stream urine samples were collected from 2190 patients. The specimens were cultured and the isolates were identified using standard microbiological techniques. The antibiotic susceptibilities of the isolates were also determined. Of 2190 specimens, 591 (27\%) showed significant growth upon culture. Approximately $84.1 \%(497 / 591)$ of the 591 patients with UTI were females, most of which belonged to the 21-30 age group (206). The males accounted for $15.9 \%$ (94/591) UTI cases. Most of the male patients belonged to the 21-30 age group (34). The lowest incidence of urinary tract infections was seen among the 13-20 years age group. Throughout this study males accounted for only $16 \%$ of all UTI cases. Esherichia coli was the most predominant isolate, $53.8 \%$ followed by Klebsiella pneumoniae $22.4 \%$ and Pseudomonas aeruginosa $7.6 \%$. All isolates were fully sensitive to ofloxacin, and more than $94 \%$ were sensitive to cefuroxime. Apart from group D Streptococcus, the overall response to ampicillin by all isolates was less than $15 \%$. The prevalence of multi-resistant Pseudomonas aeruginosa in community-acquired urinary tract infections is increasing. All Pseudomonas aeruginosa isolates were fully susceptible to cefuroxime and ofloxacin. It is recommended that cefuroxime and ofloxacin or both are used in the blind treatment of urinary tract infection while awaiting the culture and sensitivity results. Concurrent with the necessary shift in the prescription pattern, attention should be paid to restriction of antibiotic abuse in the community to retard development of further drug resistance.
\end{abstract}

\section{Introduction}

Broadly speaking, urinary tract infections (UTI) refer to infections occurring anywhere along the urinary tract from the perinephric fascia to the urethral meatus. Urinary tract infections are common in general practice ${ }^{\mathbf{1 , 2}}$ and have important economic consequences ${ }^{2}$. UTI is one of the most common diseases encountered in clinical practice today. Urinary tract infection is not only common but the range of clinical effects varies from asymptomatic bacteriuria to acute pyelonephritis ${ }^{3}$. Bacterial infections of the urinary tract have been reported in hospital and community situations ${ }^{\mathbf{4 , 5}}$. In the community the prevalence has been reported in all age groups and in both sexes. In women between the ages of 16-35 years, the prevalence of urinary tract infection is approximately $20 \%^{6}$, and the major risk factors in this age group appear to be sexual intercourse and the use of the diaphragm and spermicide $^{\mathbf{7 , 8}}$. Among young men who develop uncomplicated UTI, homosexuality as a result of exposure of the urethra to Escherichia coli (E.coli) during receptive rectal intercourse, lack of circumcision and human immuno-defiency virus (HIV) infection, are important risk factors ${ }^{\mathbf{9}, 10}$. The prevalence of bacteriuria is approximately $1 \%$ in schoolgirls, but rare in elementary school-age boys $^{11}$. In the elderly the incidence of UTI increases sharply in both sexes, and rates of $17 \%$, $55 \%$ and $15 \%-31 \%$ have been reported in women and men ${ }^{12,13}$.

The disorders are generally treated empirically with antibiotics. It is useful for GPs to be aware of the locally prevalent strains of uropathogens and their sensitivity pattern in order to decide on their choice of antibiotics. This knowledge might help primary care physicians who were often guilty of excessive and inappropriate use of antibiotics ${ }^{14}$. The judicious use of antibiotics requires accurate data on antimicrobial susceptibility which may vary in time and place. Regular surveillance is therefore necessary. 
Urinary pathogens, especially from community patients, have been known to include strains that are resistant to many of the commonly used antibiotics. ${ }^{15}$ Data on the patterns of resistance in most developing countries is inadequate. In Kashmir little is known about the resistance patterns of urinary pathogens isolated from patients living in different communities. This study reviews the susceptibility profiles of urinary isolates from patients who were seen in a general practice in Srinagar, Kashmir over a period of 24 months.

\section{Material and Methods}

Study population: Urine samples were collected from a total of 2190 patients suspected of urinary tract infection at Al-Haram diagnostic, research and training center, Srinagar, Kashmir, India during the period of April 2007 - March, 2009. The subjects were made up of 1890 females and 300 males. All these persons were outpatients visiting different private practioners and private hospitals in Srinagar city. Patients who have been on antibiotics for at least three days were excluded from the study.

Sterilization of media and materials: The media used were cysteine lactose electrolyte deficient (CLED) media, Sheep Blood Agar (SBA) and Muller Hinton Agar supplied by Oxoid Limited. All glassware were washed with detergent and rinsed with water, then allowed to dry. The glassware were later wrapped in aluminum foil and sterilized in a hot air oven at $160^{\circ} \mathrm{C}$ for $3 \mathrm{hrs}$. Media were sterilized by autoclaving at $121^{\circ} \mathrm{C}$ for 15 mins.

Urine collection: Patients with suspected UTI were instructed in the method of midstream urine (MSU) collection with aseptic precaution (posters in the collection areas re-inforce the method). Specimens were collected in wide mouth screw-on-top containers and stored at $4^{\circ} \mathrm{C}$ until transported to our laboratory in Eight-quart Coleman coolers. Quantitative bacteriologic cultures were performed according to standard laboratory procedures ${ }^{\mathbf{1 6}}$.

Culturing of urine sample: All samples were processed within one hour after arriving at the laboratory. A standard calibrated platinum wire loop delivering $0.001 \mathrm{ml}$ of uncentrifuged urine was used to inoculate sheep blood agar (SBA) and cysteine lactose electrolyte deficient (CLED) media. These plates were incubated aerobically at $35-37^{\circ} \mathrm{C}$ for $18-24$ hours. Specimens of urine containing $>10^{5}$ organisms per $\mathrm{ml}$ of a single species were considered as having significant bacteriuria. Wet preparations were made from centrifuged samples and casts, leukocytes and erythrocytes per high power field were looked for. A rapid dipstick (Combur 10 test, Boehringer Manheim, Diagnostics and Biochemicals, East Sussex, United Kingdom) was also used to detect pyuria via leukocyte esterase activity ${ }^{17}$.

Bacterial identification: Identification of organisms was based on gram reaction, morphology, and biochemical characteristics. In addition isolates were also identified by using commercially available API 20 E kit (BioMerieux, France).

Antibiotic sensitivity test: Antibiotic susceptibility testing was carried out using the Kirby-Bauer disc diffusion technique on Muller-Hinton agar and commercial antibiotic discs (Oxoid, United Kingdom) were used for antimicrobial testing ${ }^{\mathbf{1 8}}$. The antibiotic discs used were: Ampicillin $(10 \mu \mathrm{g})$,

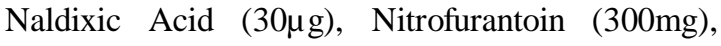

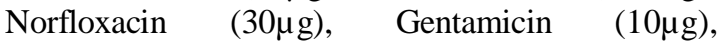
Trimethoprim-Sulphamethoxazole $(1.25 / 23.75 \mu \mathrm{g})$, Ceftazidime $(30 \mu \mathrm{g})$, Ciprofloxacin $(5 \mu \mathrm{g})$, Cefuroxime $(30 \mu \mathrm{g})$ and ofloxacin $(5 \mathrm{mg})$. The antibiotic disc impregnated culture plates were incubated at $37^{\circ} \mathrm{C}$ for overnight. The diameter of the zone of inhibition was measured and recorded as resistant or susceptible according to the National Committee for Clinical Laboratory Standards (NCCLS) interpretative criteria ${ }^{\mathbf{1 9}}$.

\section{Results}

A total of 2190 urine specimens were submitted for culture and sensitivity testing, of which 1890 $(86.3 \%)$ were obtained from females and 300 (13.7\%) were from males. Of 2190 specimens, 591 (27\%) showed significant growth upon culture.

Table I Portrays the distribution of patients with UTI by age and gender. Approximately $84.1 \%$ $(497 / 591)$ of the 591 patients with UTI were females, most of which belonged to the 21-30 years age group (206) followed by 31-40 years (106) and 41-50 years (95) respectively. The males accounted for $15.9 \%$ (94/591) UTI cases. Most of the male patients belonged to the 21-30 years age group (34) followed by 31-40 years (17) and 41-50 (15) respectively. The lowest incidence of urinary tract infections was seen among the 13-20 years age group closely followed by the $0-12$ years old patients. Throughout this study males accounted for only $16 \%$ of all UTI cases seen in this general practice.

Table I: Distribution of Patients with UTI by age and sex

\begin{tabular}{lccccccc}
\hline Sex & \multicolumn{7}{c}{ Age group in years } \\
& $0-12$ & $13-20$ & $21-30$ & $31-40$ & $41-50$ & $>50$ & Total \\
\hline Male & 12 & 4 & 34 & 17 & 15 & 12 & 94 \\
Female & 19 & 21 & 206 & 106 & 95 & 50 & 497 \\
\hline Total & 31 & 25 & 240 & 123 & 110 & 62 & 591 \\
\hline
\end{tabular}


Table II depicts the distribution of Uropathogens and their resistance pattern to various antimicrobial agents. E.coli was the most frequent isolate, 53.8\% (318/591), followed by K.pneumoniae $22.4 \%$ (132/591), Pseudomonas aeruginosa $7.6 \%$ (45/591), Proteus mirabilis 4.0\% (24/591), Enterobacter spp. 3.0\% (18/591), Acinetobacter spp. 2.7\% (16/591) and group D Streptococcus $6.5 \%(38 / 591)$. None of the specimens yielded polymicrobial cultures. All isolates except two E.coli and one Klebsiella pneumoniae were fully sensitive to ofloxacin, and more than $94 \%$ were sensitive to cefuroxime. A part from group D Streptococcus, the overall response to ampicillin by all isolates was less than 15\%. All Klebsiella pneumoniae isolates were resistant to Ampicillin. P.aeruginosa isolates were susceptible to cefuroxime, ceftazidime and ofloxacin.

Table II: Distribution of Uropathogens and their Resistance Pattern to various antimicrobial agents

\begin{tabular}{|l|c|c|c|c|c|c|c|c|c|c|c|}
\hline \multicolumn{9}{|l|}{} & \multicolumn{6}{l|}{ Percent of resistant isolates against } \\
\hline Organism & $\begin{array}{c}\text { No. \& \% } \\
\text { of Isolates }\end{array}$ & AMP & Co-Tri & $\begin{array}{c}\text { CX } \\
\text { M }\end{array}$ & Ofl & CAZ & CIP & GM & NA & NOR & NIT \\
\hline Escherichia coli & $318(53.8)$ & 75.2 & 62.2 & 1.3 & 0.6 & 48.1 & 15.2 & 58.5 & 67.2 & 42.1 & 36.2 \\
\hline Klebsiella pneumoniae & $132(22.4)$ & 100 & 44.4 & 2.5 & 0.7 & 37.1 & 22.1 & 17.4 & 55.5 & 34.4 & NT \\
\hline Pseudomonas aeruginosa & $45(7.6)$ & NT & NT & 5.2 & 0 & 14.1 & 26.8 & 15.8 & NT & 58.2 & NT \\
\hline Proteus mirabilis & $24(4.0)$ & 76.1 & 66.1 & 13.8 & 0 & 38.8 & 18.3 & 59.1 & 68.8 & 34.4 & NT \\
\hline Enterobacter spp. & $18(3.0)$ & 88.1 & 69.0 & 6.6 & 0 & 48.6 & 17.7 & 55.0 & 66.9 & 33.3 & 49.9 \\
\hline Acinetobacter spp. & $16(2.7)$ & 90.1 & 74.3 & 8.3 & 0 & 49.0 & 15.5 & 56.1 & 76.3 & 45.6 & 18.2 \\
\hline Group D Streptococcus & $38(6.5)$ & 15.1 & NT & 2.6 & 0 & NT & 40.9 & 70.4 & 44.1 & 35.7 & 29.7 \\
\hline
\end{tabular}

$\mathrm{AMP}=$ ampicillin, Co-Tri=Cotrimoxazole, $\mathrm{CXM}=$ Cefuroxime, Ofl=ofloxacin, $\mathrm{CAZ}=$ ceftazidime, $\mathrm{CIP}=$ ciprofloxacin, $\mathrm{GM}=$ gentamicin, $\mathrm{NA}=$ nalidixic acid, $\mathrm{NOR}=$ norfloxacin, $\mathrm{NIT}=$ nitofurantion, $\mathrm{NT}=$ not tested

\section{Discussion}

The study was undertaken in Srinagar city to determine the antimicrobial susceptibility pattern of urinary tract infection as well as to evaluate the bacterial agents involved in this UTI. The predominance of Escherichia coli among the causative agents of urinary tract infection in this report supports the view ${ }^{20-23}$, that E. coli even now is the most frequent cause of the infection. However, the incidence of infection due to this organism in this series $(54 \%)$ was much lower than that reported by $\operatorname{Erwa}^{24}(70.9 \%)$ and Mahgoub et $\mathrm{al}^{25}$, (75\%). Nevertheless, its incidence compares well with that recorded in other reports ${ }^{23,26,27}$. However, our incidence was higher than that reported by Aiyegoro et $\mathrm{al}^{28},(52 \%)$.

The results of this survey also showed both E.coli and Klebsiella pneumoniae as the main causes of urinary tract infection in Srinagar where they accounted for $76 \%$ of the isolates. It corroborated well with that of Shamweel and Mubaraki ${ }^{23}, 2011$; Shamweel and Farooque ${ }^{26}$, 1995; Ahmed and Ragaa $^{27}$, 1988, where E. coli and Klebsiella pneumoniae accounted for approximately $70 \%$ of the isolates respectively. It is therefore evident that the predominant organisms associated with UTI are gram-negative bacteria belonging to the family Enterobacteriaceae. These organisms are part of the normal flora of the gastrointestinal tract. The short female urethra and the proximity of the anus to the urethra make females more prone to bacterial infections, due to colonization of the anterior urethra and the vaginal introitus. This may then partly account for the higher prevalence and incidence of UTI in females. Also, other predisposing factors of UTI include clinical conditions as pregnancy, prostatic disease, hydronephrosis, urethro-pelvic stenosis and constipation $^{29,30}$.

Several reports have shown that the incidence rates for UTI vary with age and sex, with a higher incidence occurring in those over 20 years of age $^{\mathbf{8 , 3 0 , 3 1}}$. Our results indicate that the incidence in adult females was about 6 times higher than males of similar age. The great majority of males with UTI in this study, 96\%, had indwelling urethral catheters due to prostatic disease and only came to this practice because the hospital was always overcrowded or little far away. These men were either unfit for surgery, refused surgery or there was a long waiting list for surgery ${ }^{32}$.

International guidelines for treatment of acute uncomplicated urinary infection have updated the Infectious Diseases Society of America (IDSA) treatment guideline of $1999^{33}$. The updated guidelines consider empiric antimicrobial therapy for acute cystitis in the context of the increasing global resistance observed in E. coli ${ }^{34}$. The term "collateral damage" is used to describe the propensity of an antimicrobial to induce resistance in gut flora which may then compromise infection management beyond cystitis. For empiric treatment of acute uncomplicated cystitis recommended first line agents are TMP/SMX or TMP by itself for 3 days if the local prevalence of resistance is less than $20 \%$, nitrofurantoin for 5 days, pivmecillinam 
for 3-7 days, or fosfomycin as a single dose. Three of these agents like nitrofurantoin, pivmecillinam, and fosfomycin are currently indicated only for acute cystitis, and are unique classes of antimicrobials without cross-resistance with other agents. Fluoroquinolones given for three days may be $5 \%$ to $10 \%$ more effective in clinical trials than some of the recommended first line regimens, but are discouraged for use as first line therapy because of the propensity for "collateral damage." For oral treatment of acute nonobstructive pyelonephritis, ciprofloxacin, ofloxacin or levofloxacin are recommended $^{33}$. TMP/SMX or TMP are effective alternate oral agents if the infecting organism is known to be susceptible. When TMP/SMX is used empirically, an initial parenteral dose of an aminoglycoside or ceftriaxone is suggested for coverage of potential TMP/SMX resistant organisms pending culture results. Empiric parenteral therapy for pyelonephritis is little changed from the 1999 guidelines. Suggested regimens include an aminoglycoside with or without ampicillin, a third generation cephalosporin, or a fluoroquinolone.

The antibiotic in-vitro susceptibility testing showed that co-trimoxazole and ampicillin are unlikely to be effective in treating patients with Urinary tract infection due to E. coli, Klebsiella pneumoniae and Proteus mirabilis in this community as these organisms showed relatively low sensitivity to these drugs. Improper use of antibiotics often facilitates the selection of resistant bacterial strains. The more antibiotics are used in the community the more likely it is that resistant strains will be selected and maintained in that environment ${ }^{35}$. In Srinagar self mediation with easy-to-obtain over the counter drugs such as co-trimoxazole and ampicillin, are very common. The inadequate dosage of these antibiotics used in self-medication, along with the wide spread use in animal feeds of antibiotics to improve livestock are important contributors to the development of resistant strains to these drugs in the community.

Srinagar city is heavily populated and majority of the hospitals are usually heavily attended and sometimes the doctor only spends a few hours there and leaves, thus putting tremendous financial pressure on patients who desperately need help to visit the general practitioners. The high resistance rate of uropathogens to some of the commonly used oral antimicrobial agents as seen in this study, has influenced the introduction of the use of relatively new antibiotics such as cefuroxime and ofloxacin into the country, which may produce higher cure rates as evidenced by in-vitro testing against more than $94 \%$ of urinary isolates in this study.
The high frequency of single and multiple antibiotic resistance in this community should cause alarm. The ease of procuring antibiotics in these areas without a prescription can result in uncontrolled self-medication. Also, the absence of antibiotic prescribing policies and in adequate information on patterns of bacterial resistance, may all contribute to the emergence of resistant strains. It can be concluded from this study that $E$. coli and Klebsiella pneumoniae were the main pathogens isolated and that the majority of pathogens were sensitive in vitro to ofloxacin and cefuroxime or both. It is recommended that ofloxacin or cefuroxime, or both, are used in the empirical treatment of UTIs, following the submission of MSU specimens for culture, while awaiting the culture and sensitivity results in view of high prevalence of UTI in Kashmir. It is also recommended to monitor continuously the pattern of urinary pathogens, from the community at large, and their resistance patterns to guide the empirical treatment of patients in the future.

\section{References}

1. Lim TO. Content of general practice. Med J Malaysia. 1991;46(2):155-62.

2. Patton JP, Nash DB, Abrutyn E. Urinary tract infection: economic consideration. Med Clin North Am. 1999;75(2):495-513.

3. Ahmed S, Rashid HU. Urinary tract infection in adults: A review. Bangladesh Renal J. 1996; 15: 23-31.

4. Raz R. Antimicrobial resistance of urinary isolates in the community and its relation to antibiotic use. Isr $\mathbf{J}$ Med Sci 1993; 29: 207-210.

5. Adjei O. Antibiotic susceptibility of urinary pathogens from inpatients in Kumasi, Ghana. Trop Doctor 1993; 23: 29-30.

6. Valkenburg HA. Epidemiology of urinary tract infections. Europ Urol 1987; 13 Suppl 1: 5-8.

7. Hooton TM, Hillier S, Johnson C, Roberts PL, Stamm WE. Escherichia coli bacteriuria and contraceptive methods. JAMA 1991; 265: 64-67.

8. Strom BL, Collins M, West SL, Kreisberg J, Weller S. Sexual activity, contraceptive use and other risk factors for symptomatic and asymptomatic bacteriuria. Ann Intern Med 1987; 107: 816- 823.

9. Spach DH, Stapleton AE, Stamm WE. Lack of circumcision increases the risk of urinary tract infection in young men. JAMA 1992; 267: 679-682.

10. Barnes RC, Daifuku R, Reddy RE, Stamm WE. Urinary tract infections in sexually active homosexual men. Lancet 1986; 2: 171-174.

11. Gillenwater JY, Harrison RB, Kunin CM. Natural history of bacteriuria in school girls: A long term casecontrol study. N Engl J Med 1979; 301: 369-372. 
12. Emori TG, Banerjee SN, Culver DH, Gaynes RP, Horan TC, Jarvis WR et al. Nosocomial infections in elderly patients in the United States, 1986-1990. Am J Med 1991; 91 Suppl 3B: 289S-293S.

13. McClure CL. Common infections in the elderly. Am Fam Phys 1992; 45: 2691- 2698.

14. Teng CL, Achike FI, Phua KL, et al. Modifying antibiotic prescribing: The effectiveness of academic detailing plus information leaflet in a Malaysian primary care setting. Med J Malaysia. 2006; 61(3): 323-31.

15. Young HK, Jesudason MV, Keshi G, Amyes SG Trimethoprim resistance amongst urinary pathogens in South India. J Antimicrob Chemother 1986; 17: 615-621.

16. Pezzlo M. Processing and interpretation of urine cultures. In: Isenberg $\mathrm{HD}$, editors. Essential Procedures for Clinical Microbiology. Washington DC: American Society for Microbiology; 1998. p. 95-101.

17. Kusumi RK, Grover PJ, Kunin CM. Rapid detection of pyuria by leukocyte esterase activity. JAMA 1981; 245: 1653-1655.

18. Bauer AW, Kirby WMM, Sherris JC, Jurek M. Antibiotic Susceptibility testing by a standardized single method. Am J Clin Pathol 1996; 45 : 493-6.

19. Performance standards for antimicrobial susceptibility testing. Tenth informational supplement. National Committee for Clinical Laboratory Standards (NCCLS), 2000: M100-S10 (M2): 14-21.

20. Pulverers G, Cho C H, Spikeman C H. Etiology of pyogenic urinary tract infections in the region in Bayer symposium III. Bacterial infections, changes in their casual agents, trends and possible basis, pp 25-29; 1971. Springer-verlag, Berlin, Heidelberg. New York.

21. Akbar DH. Urinary tract infection. Diabetics and nondiabetic patients. Saudi Medical Journal 2001; Vol. 22 (4): 326-329.

22. Rafay AM. Urinary tract infections in general practice in a rural community in South Trinidad. Saudi Medical Journal 2003; Vol. 24 (3): 261-264.

23. Shamweel A, Mubaraki AM. Antimicrobial Susceptibility of Gram Negative Organisms in Urine Cultures at Armed Forces Hospital in Saudi Arabia. Dis J Pak 2011; 20(4):357-360.

24. Erwa H H. Escherichia coli in urinary tract infections in Sudanese patients. Trops \& Geog. Med. 1972; 24: 60-65.
25. Mahgoub E, Chowdhury H, Jamjoom GA, Kambal AM.The pattern of bacteriuria of urinary tract infection at king Abdul Aziz teaching Hospital, Riyadh. Proceedings of the $7^{\text {th }}$. Saudi Medical meeting 1982; 221-4.

26. Shamweel A, Ahmad F. Urinary tract infection at a specialist hospital in Saudi Arabia. Bangladesh Med. Res. Counc. Bull. 1995; 21(3):95-98.

27. Ahmed T, Eltahawy Ragaa MF.Khalaf. Urinary tract infection at University hospital in Saudi Arabia: incidence, microbiology, and antimicrobial susceptibility. Annals of Saudi Medicine 1988; 8(4): 261-266.

28. Aiyegoro O A, Igbinosa O O, Ogunmwonyi I N, Odjadjare E E1, Igbinosa O E, Okoh A I. Incidence of urinary tract infections (UTI) among children and adolescents in Ile-Ife, Nigeria. Afr. J. Microbiol. Res. July 2007; 13-19.

29. O'Regan S, Yazbeck S, Schick E. Constipation bladder instability, urinary tract infection syndrome. Clin Nephrol 1985; 23: 152-155.

30. Stamm WE, Stapleton AE. Approach to the patient with urinary tract infection. In: Gorbach SL, Bartlett JG, Blacklow NR, editors. Infectious Diseases. 2nd ed. Philadelphia (USA): WB Saunders Company; 1998. p. 943-954.

31. Ako-Nai AK, Kassim OO, Adenitan MO, Taiwo O. A study of urinary tract infections at ILE-IFE, Nigeria. East Afr Med J 1993; 70: 10-14.

32. Orrett FA, Premanand N. Bacteruria in outpatients with chronic indwelling urethral catheters. Med Sci Res 1993; 21: 333-334.

33. Gupta K, Hooton TM, Naber KG, et al.: International clinical practice guidelines for the treatment of acute uncomplicated cystitis and pyelonephritis in women: a 2010 update by the Infectious Diseases Society of America and the European Society for Clinical Microbiology and Infectious Diseases. Clin Infect Dis. 2011;52:561-4.

34. Schito GC, Naber KG, Botto H, et al.: The ARESC study: an international survey on the antimicrobial resistance of pathogens involved in uncomplicated urinary tract infections. Int J Antimicrob Agents 2009; 34: 407-13.

35. Ringertz S, Bellete B, Karlsson I, Ohman G, Gedebou M, Kronvall $G$ et al. Antibiotic susceptibility of Escherichia coli isolates from in-patients with urinary tract infections in hospitals in Addis Ababa and Stockholm. Bull. WHO 1990; 68: 67-68. 